\title{
AVALIAÇÃO DA ATENÇÃO PRIMÁRIA À SAÚDE DE CRIANÇAS NA ÓTICA DOS PAIS E CUIDADORES
}

\author{
EVALUATION OF PRIMARY HEALTH CARE OF CHILDREN \\ FROM THE VIEWPOINT OF PARENTS AND CAREGIVERS
}

Bianca Fontana Aguiar', Noeli Maria Rodrigues Alves Santos Hack², Leandro Rozin ${ }^{3}$

\section{RESUMO}

Fragilidades na atenção à saúde da criança, como a falta de estrutura dos serviços e a prevalência de internações pediátricas, podem ser evitadas com serviços de Atenção Primária à Saúde (APS) qualificados, eficientes e resolutivos. Objetivou-se aplicar o Instrumento de Avaliação da APS aos pais e responsáveis de crianças menores de dois anos e avaliar os atributos da APS. Estudo de campo, com abordagem quantitativa, realizado em duas unidades de saúde (US) do município de Curitiba/ PR em 2015, por meio do Instrumento de Avaliação da Atenção Primária infantil, sendo realizadas 100 entrevistas. Para análise utilizou-se estatística descritiva. Em relação à afiliação, $100 \%$ dos usuários levam seu filho (a) no serviço de saúde; na utilização, $74 \%$ vão a US antes de outros serviços; na acessibilidade, $54 \%$ dos entrevistados levaram a criança à US e 0 serviço atendeu no dia; na Longitudinalidade, $48 \%$ sempre o mesmo médico e enfermeiro atendem a criança; na coordenação, $88 \%$ relataram que a sua criança não consultou especialistas; no sistema de informação, $54 \%$ afirmaram que o prontuário está disponível na US; nos serviços disponíveis, $90 \%$ das US têm vacinas disponíveis; quanto aos serviços prestados, $75 \%$ foram orientados em como manter sua criança saudável; na orientação familiar, $48 \%$ afirmaram que o médico se reuniria com outros membros da família da criança; já na orientação comunitária, 56\% recebem visita domiciliar. A avaliação dos pais/cuidadores, quanto ao serviço da APS, contribui para subsidiar melhorias na atenção prestada à saúde da criança e qualificar a assistência dos profissionais nas unidades de saúde.

Descritores: Atenção Primária à Saúde; Pediatria; Centros de Saúde.

\section{ABSTRACT}

Fragilities in health care of children, the lack of structure of services and the prevalence of pediatric hospitalizations can be avoided with Primary Health Care Services (APS) qualified, efficient and resolute. Aimed to apply the APS Assessment Tool to parents and guardians of children under two years and evaluate the attributes of APS. Field of study, with a quantitative approach, carried out in two health units (US) in the city of Curitiba / PR in 2015, through the Primary Care Children's Assessment Instrument, being carried out 100 interviews. For analysis was used descriptive statistics. Regarding membership, $100 \%$ of users take their child (a) in the health service; In use, $74 \%$ go to us before other services; accessibility, $54 \%$ of respondents took the child to the US and attended the service on the day; in longitudinality, $48 \%$ always the same doctor and nurse attending to a child; coordination, $88 \%$ reported that their child did not consult experts; in the information system, $54 \%$ said that the medical record is available in the US; the available services, $90 \%$ of US vaccines are available; as the services provided, $75 \%$ were instructed on how to keep your child healthy; in family counseling, $48 \%$ said that the doctor would meet with other child's family members; already in the community orientation, $56 \%$ receive home visits. The evaluation of the parents / carers, as the service of APS helps to subsidize improvements in attention paid to children's health and qualify the assistance of professionals in health facilities.
${ }^{1}$ Graduada em Enfermagem pela Universidade Estadual de Ponta Grossa (UEPG), Ponta Grossa, PR, Brasil.

${ }^{2}$ Graduada em Enfermagem e Obstetrícia pela Universidade Federal do Paraná (UFPR), Curitiba, PR, Brasil.

${ }^{3}$ Graduado em Enfermagem pela Faculdade de Pato Branco (FADEP), Pato Branco, PR, Brasil. 


\section{Introdução}

A atenção primária à saúde (APS), abrange ações de promoção e proteção da saúde, as quais são desenvolvidas por equipes multidisciplinares, aptas a resolver os problemas de saúde de maior incidência em cada região. O Sistema Único de Saúde (SUS) utiliza da APS para enfatizar a reorientação do modelo assistencial, a partir de um sistema universal e integrado de atenção à saúde 1 . É por meio da Estratégia de Saúde da Família (ESF) que a APS se organiza para melhor desenvolvimento do seu trabalho².

As mudanças epidemiológicas e demográficas existentes no sistema de saúde, em geral, demonstram a necessidade de implantação de estratégias inovadoras para a assistência e administração de recursos humanos e financeiros, promovendo o aperfeiçoamento do sistema de saúde 3 . A ESF auxilia os usuários no acesso aos serviços e no estreitamento com a equipe, que tem corroborado para a melhoria nos indicadores de saúde. Baseado na importância que a ESF tem demonstrado na APS é fundamental fazer a sua avaliação, a fim de apontar as suas potencialidades e fragilidades que podem servir como subsídio para gestão em saúde ${ }^{4,5}$.

Nos últimos anos, a prática de avaliação dos serviços de saúde vem ganhando destaque, e alguns pesquisadores desenvolveram um instrumento que permite mensurar a presença e a extensão dos atributos essenciais e derivados da APS, o Primary Care Assessment Tool (PCATool). Tal instrumento possui versão infantil, que permite, por meio de entrevistas com cuidadores de crianças de até dois anos de idade, identificar aspectos do funcionamento, da estrutura e do processo dos serviços ${ }^{6}$.

Ações de promoção e prevenção à saúde são prioritárias na APS, porém observa-se fragilidades como a falta de estrutura dos serviços e também a prevalência de internações pediátricas que poderiam ter sido evitadas por meio de atenção qualificada e resolutiva. Justifica-se assim a necessidade de processos avaliativos, a fim de para mensurar a qualidade da APS no país?

Na saúde da criança, espera-se a partir dos serviços de saúde, políticas e ações voltadas à população infantil, a fim de diminuir os indicadores de morbimortalidade infantil e garantir o crescimento e desenvolvimento saudável, visto que os atendimentos integrais nos primeiros anos de vida são fundamentais à manutenção de sua vida ${ }^{8}$. Sendo assim, a avaliação da APS possibilita conhecer a opinião dos cuidadores sobre 0 atendimento prestado às crianças e a identificação dos fatores positivos e negativos da atenção primária na busca de seu aperfeiçoamento.

Por entender a importância da assistência pautada nos princípios e diretrizes do SUS, o presente estudo teve como objetivo aplicar o Instrumento de Avaliação da APS aos pais e responsáveis de crianças menores de dois anos e avaliar os atributos da APS, que são eles: grau de afiliação com serviço, acesso de primeiro contato, acessibilidade, longitudinalidade, integração de cuidados, sistema de informações, integralidade dos serviços disponíveis e serviços prestados, orientação familiar e comunitária.

\section{Metodologia}

Estudo exploratório descritivo com abordagem quantitativa, desenvolvido em duas unidades de saúde, ESF, em Curitiba - Paraná. 0 município de Curitiba possui 65 unidades de saúde com 241 equipes de ESF. As duas unidades foram selecionadas por fazerem parte do campo de atuação da residência em saúde da criança e do adolescente em que estávamos inseridos.

Os participantes foram os pais/ou cuidadores das crianças de zero a dois anos de idade residentes e cadastradas nas áreas de abrangência das ESFs determinadas pelo estudo. Foram excluídos os usuários que não compareceram nas consultas de puericultura, aqueles que não souberam responder as perguntas da coleta de dados e os que não se encontravam em casa quando realizada visita domicilar.

A coleta de dados foi realizada durante as demandas de puericultura na unidade de saúde e por meio de visitas domiciliares, com uso de questionário validado no Brasil denominado de Instrumento de Avaliação da APS na versão infantil (PCATool-Brasil versão infantil), o qual foi aplicado aos pais/ou cuidadores das crianças residentes e cadastradas nas áreas de abrangência da unidade de saúde (US).

O uso deste instrumento é recomendado pelo Ministério da Saúde (MS), sendo composto por 55 itens divididos em 10 componentes relacionados aos atributos essenciais e derivados da APS: grau de afiliação com serviço de saúde, 
acesso de primeiro contato, acessibilidade, longitudinalidade, coordenação, sistema de informações, serviços disponíveis, serviços prestados, orientação familiar, orientação comunitária.

Inicialmente, foi realizado levantamento nas áreas de abrangência da unidade de saúde para identificação de crianças de 0 a 2 anos cadastradas. Pelo número de crianças menores de 1 ano ser semelhante nas duas unidades de saúde e o tempo para coleta dos dados ocorrer em dois meses, optou-se por padronizar 50 entrevistas para cada unidade. De forma aleatória, foram realizadas as visitas domiciliares, agendadas com as agentes comunitárias, procurando também alternar as micros áreas do território adscrito.

As informações foram coletadas após aprovação do comitê de ética e pesquisa, nos meses de agosto e setembro de 2015, sem recusa dos pais/cuidadores em participarem do estudo. Posteriormente esses dados foram agrupados pelo pesquisador, permitindo assim sua análise. Após agrupamento dos dados obtidos na aplicação do instrumento, estes foram transformados em informações para serem analisadas.

A análise foi baseada em estatística descritiva. As respostas do questionário estão em uma escala tipo Likert: "com certeza sim", "provavelmente sim", "provavelmente não", "com certeza não" e "não sei / não lembro". Os resultados foram apresentados em tabelas, com base em dados categóricos e numéricos.

Em todo o momento deste trabalho, se respeitaram os preceitos éticos de participação voluntária e consentida segundo Resolução 466/12, por meio do Termo de Consentimento Livre e Esclarecido, sendo este obtido de todos os participantes, antes do início da entrevista.

A realização se deu mediante autorização do Comitê de Ética em Pesquisa da FPP e autorização da Secretaria Municipal de Saúde de Curitiba-PR, sob CAAE: 44572715.3.0000.5580 e parecer de aprovação $n^{0}$ 1.174.354. Salientamos que foram seguidos rigorosamente os aspectos éticos, destacando o anonimato dos entrevistados, com base na beneficência, não-maleficência e justiça.

\section{Resultados e Discussão}

Participaram deste estudo 100 pessoas, cadastradas nas ESF selecionadas sendo os sujeitos entrevistados: mães, pais e outros cuidadores, principais responsáveis pelas crianças com idade igual ou inferior a dois anos de idade. Não houve nenhuma recusa à pesquisa.

\section{GRAU DE AFILIAÇÃO}

A (TABELA 1) mostra o grau de afiliação do usuário com o serviço de saúde.

Tabela 1 - Grau de afiliação

\begin{tabular}{|c|c|c|c|c|c|c|}
\hline \multirow[t]{2}{*}{ A- Grau de afiliação } & \multicolumn{2}{|c|}{ SIM } & \multicolumn{2}{|c|}{ NÃO } & \multicolumn{2}{|c|}{ TOTAL } \\
\hline & $N$ & $\%$ & $N$ & $\%$ & $N$ & $\%$ \\
\hline $\begin{array}{l}\text { A1- Há algum médico/enfermeiro ou serviço de } \\
\text { saúde que você leva seu filho(a) quando ele adoece. }\end{array}$ & 100 & 100 & 0 & 0 & 100 & 100 \\
\hline $\begin{array}{l}\text { A2- Há um médico/enfermeiro ou serviço de saúde } \\
\text { que conhece melhor seu filho(a)? }\end{array}$ & 97 & 97 & 3 & 3 & 100 & 100 \\
\hline $\begin{array}{l}\text { A3- Há um médico ou serviço de saúde que é mais } \\
\text { responsável pelo atendimento de seu filho(a)? }\end{array}$ & 97 & 97 & 3 & 3 & 100 & 100 \\
\hline
\end{tabular}

Fonte: dados da pesquisa, 2016.

No atributo grau de afiliação observou-se que $100 \%$ dos usuários levam seus filhos àAPS quando adoecem, apontando maior confiança e segurança no serviço. Podendo-se atribuir isso à proximidade do serviço à casa do usuário, à relação mais próxima com a equipe e a eficácia do atendimento. Quanto aos itens que avaliam se existe algum profissional de saúde que 
conhece melhor a criança e se existe algum médico responsável pelo atendimento, $97 \%$ dos usuários responderam que sim para ambos os itens, reforçando que existe a proximidade entre a APS e seus usuários. Um estudo realizado por OLIVEIRA, comprova a existência da adesão da população em relação à ESF, tendo o reconhecimento do usuário por um profissional ou serviço que o mesmo acredita ser de referência sempre que é preciso $0^{9,10}$.

\section{UTILIZAÇÃO}

Observa-se (TABELA 2) o acesso de primeiro contato relacionado a utilização do serviço de saúde pelo usuário.

Tabela 2 - Acesso de primeiro contato - utilização

\begin{tabular}{c|c|c|c|c|c|c|c|c}
\hline $\begin{array}{c}\text { B-Acesso de primeiro } \\
\text { contato - utilização }\end{array}$ & \multicolumn{2}{|c|}{ Com certeza sim } & \multicolumn{2}{|c|}{ Provavelmente sim } & \multicolumn{2}{|c|}{ Provavelmente não } & \multicolumn{2}{|c}{ TOTAL } \\
\hline & $N$ & $\%$ & $N$ & $\%$ & $N$ & $\%$ & $N$ & $\%$ \\
\hline $\begin{array}{c}\text { B1- Quando sua criança } \\
\text { necessita de uma consulta } \\
\text { de revisão você vai à } \\
\text { unidade de saúde antes de } \\
\text { procurar outro serviço? }\end{array}$ & 74 & 74 & 18 & 18 & 8 & 8 & 100 & 100 \\
\hline $\begin{array}{c}\text { B2- Quando sua criança } \\
\text { tem um novo problema de } \\
\text { saúde você vai à unidade } \\
\text { de saúde antes de procurar } \\
\text { outro serviço? }\end{array}$ & 53 & 53 & 37 & 37 & 10 & 10 & 100 & 100 \\
\hline $\begin{array}{c}\text { B3- Quando sua criança } \\
\text { tem que consultar um } \\
\text { médico especialista o } \\
\text { seu médico/enfermeiro/ } \\
\text { unidade de saúde } \\
\text { tem que encaminhar } \\
\text { obrigatoriamente? }\end{array}$ & 34 & 34 & 53 & 53 & 13 & 13 & 100 & 100 \\
\hline
\end{tabular}

Fonte: dados da pesquisa, 2016.

O atributo acesso de primeiro contato - utilização demonstra que os usuários utilizam em sua maioria a APS, além de identificarem o serviço de saúde como referência ao atendimento infantil e a ESF compõe o local de primeira escolha dos cuidadores quando a criança necessita de cuidados de saúde ${ }^{11,6}$. Observa-se que $74 \%$ dos entrevistados, responderam que procuram a US para levar sua criança antes de procurar outro serviço, além de que $53 \%$ voltariam na unidade caso a criança tivesse um novo problema de saúde, considerando uma boa recepção e confiança no serviço prestado. Mostrase que a prática do acolhimento no atendimento inicial dos usuários, realizada pela equipe multiprofissional, torna-se um elemento de grande valia, estreitando as relações entre os profissionais e a população ${ }^{12}$. Segundo HAGGERTY, o contato de acesso é ideal para verificar ações de primeiro cuidado, sendo relevante nas reformas de acessibilidade de alguns países, como o Canadá ${ }^{13}$.

\section{ACESSIBILIDADE}

No que se refere à acessibilidade, encontra-se descrito na TABELA 3. 
Tabela 3 - Acesso de primeiro contato - acessibilidade

\begin{tabular}{|c|c|c|c|c|c|c|c|c|c|c|c|c|}
\hline \multirow[t]{2}{*}{$\begin{array}{l}\text { C-Acesso de } \\
\text { primeiro contato - } \\
\text { acessibilidade }\end{array}$} & \multicolumn{2}{|c|}{$\begin{array}{c}\text { Com certeza } \\
\text { sim }\end{array}$} & \multicolumn{2}{|c|}{$\begin{array}{l}\text { Provavelmente } \\
\text { sim }\end{array}$} & \multicolumn{2}{|c|}{$\begin{array}{c}\text { Provavelmente } \\
\text { não }\end{array}$} & \multicolumn{2}{|c|}{ Não lembro } & \multicolumn{2}{|c|}{$\begin{array}{c}\text { Com certeza } \\
\text { não }\end{array}$} & \multicolumn{2}{|c|}{ TOTAL } \\
\hline & $\mathrm{N}$ & $\%$ & $\mathrm{~N}$ & $\%$ & $\mathrm{~N}$ & $\%$ & $\mathrm{~N}$ & $\%$ & $\mathrm{~N}$ & $\%$ & $\mathrm{~N}$ & $\%$ \\
\hline $\begin{array}{c}\text { C1 - Quando a } \\
\text { unidade de saúde } \\
\text { está aberta e } \\
\text { sua criança fica } \\
\text { doente, alguém } \\
\text { deste serviço a } \\
\text { atende no mesmo } \\
\text { dia? }\end{array}$ & 54 & 54 & 40 & 40 & 5 & 5 & 1 & 1 & - & - & 100 & 100 \\
\hline $\begin{array}{l}\text { C2- Você tem que } \\
\text { esperar muito } \\
\text { tempo ou fala com } \\
\text { muitas pessoas } \\
\text { para marcar } \\
\text { consulta na } \\
\text { unidade de saúde? }\end{array}$ & 29 & 29 & 52 & 52 & 17 & 17 & 1 & 1 & 1 & 1 & 100 & 100 \\
\hline $\begin{array}{l}\text { C3- é fácil marcar } \\
\text { hora para consulta } \\
\text { de “ revisão } \\
\text { da criança” na } \\
\text { unidade de saúde? }\end{array}$ & 29 & 29 & 52 & 52 & 17 & 17 & 1 & 1 & 1 & 1 & 100 & 100 \\
\hline $\begin{array}{c}\text { C4- Quando você } \\
\text { chega à unidade } \\
\text { de saúde, você tem } \\
\text { que esperar mais } \\
\text { de } 30 \text { min para a } \\
\text { consulta? }\end{array}$ & 29 & 29 & 52 & 52 & 17 & 17 & 1 & 1 & 1 & 1 & 100 & 100 \\
\hline $\begin{array}{l}\text { C5- é difícil para } \\
\text { você conseguir } \\
\text { atendimento } \\
\text { médico para sua } \\
\text { criança na unidade } \\
\text { de saúde quando } \\
\text { você pensa ser } \\
\text { necessário? }\end{array}$ & 25 & 25 & 56 & 56 & 17 & 17 & 1 & 1 & 1 & 1 & 100 & 100 \\
\hline $\begin{array}{c}\text { C6- Quando a } \\
\text { unidade está } \\
\text { aberta você } \\
\text { consegue } \\
\text { aconselhamento } \\
\text { pelo telefone se } \\
\text { precisar? }\end{array}$ & 11 & 11 & 61 & 61 & 25 & 25 & 2 & 2 & 1 & 1 & 100 & 100 \\
\hline
\end{tabular}

Fonte: dados da pesquisa, 2016.

A acessibilidade constitui os elementos estruturais da APS e implica o acesso do serviço a cada novo problema ou novo episódio de um problema pelo qual as pessoas buscam atenção à saúde. Conforme análise dos resultados, 54\% confirmaram que quando a criança necessitou de atendimento foram atendidos no mesmo dia, porém identificamos a insatisfação na maioria dos cuidadores, 52\% (52) com relação ao tempo de espera para conseguir uma consulta e ser atendido o que corrobora com BRAZ e FRACOLLI que salientam a existência de alguns obstáculos que ainda impedem a utilização dos pais ou cuidadores nos serviços de saúde destinados ao atendimento de suas crianças ${ }^{11,4}$. 
Este atributo é avaliado em alguns itens, que consideram a disponibilidade da equipe para o atendimento ao usuário, como em horários fora do atendimento normal da US, como períodos noturnos e finais de semana, e o atendimento telefônico, visualizamos que $(56 \%)$ caso precisassem possivelmente conseguiriam aconselhamento pelo telefone. Destaca-se que os serviços da APS do município de Curitiba apresentam atendimento diariamente de segunda a sexta-feira, e possuem também serviços de urgências médicas, que atendem a demanda inclusive nos horários em que as unidades não estão abertas ${ }^{14}$.

\section{LONGITUDINALIDADE}

O atributo de Longitudinalidade, ou também continuidade do cuidado é observado na TABELA4.

Tabela 4 - Longitudinalidade

\begin{tabular}{|c|c|c|c|c|c|c|c|c|c|c|c|c|}
\hline \multirow[t]{2}{*}{ D- Longitudinalidade } & \multicolumn{2}{|c|}{$\begin{array}{l}\text { Com certeza } \\
\text { sim }\end{array}$} & \multicolumn{2}{|c|}{$\begin{array}{l}\text { Provavelmente } \\
\text { sim }\end{array}$} & \multicolumn{2}{|c|}{$\begin{array}{c}\text { Provavelmente } \\
\text { não }\end{array}$} & \multicolumn{2}{|c|}{ Não lembro } & \multicolumn{2}{|c|}{$\begin{array}{c}\text { Com certeza } \\
\text { não }\end{array}$} & \multicolumn{2}{|c|}{ TOTAL } \\
\hline & $\mathrm{N}$ & $\%$ & $N$ & $\%$ & N & $\%$ & N & $\%$ & N & $\%$ & N & $\%$ \\
\hline $\begin{array}{l}\text { D1 - Quando você vai } \\
\text { à unidade de saúde } \\
\text { é o mesmo médico } \\
\text { ou enfermeiro que } \\
\text { atende sua criança? }\end{array}$ & 48 & 48 & 36 & 36 & 14 & 14 & - & - & 2 & 2 & 100 & 100 \\
\hline $\begin{array}{l}\text { D2- Se você tiver } \\
\text { uma pergunta } \\
\text { sobre a saúde de } \\
\text { sua criança, pode } \\
\text { telefonar e falar com } \\
\text { o médico/enfermeiro } \\
\text { que melhor conhece } \\
\text { sua criança? }\end{array}$ & 52 & 52 & 39 & 39 & 9 & 9 & - & - & - & - & 100 & 100 \\
\hline $\begin{array}{l}\text { D3- Você acha que o } \\
\text { médico/enfermeiro } \\
\text { entende o que você } \\
\text { diz ou pergunta? }\end{array}$ & 48 & 48 & 50 & 50 & 2 & 2 & - & - & - & - & 100 & 100 \\
\hline $\begin{array}{l}\text { D4- O médico/ } \\
\text { enfermeiro responde } \\
\text { suas perguntas de } \\
\text { maneira que você } \\
\text { entende? }\end{array}$ & 58 & 58 & 40 & 40 & 2 & 2 & - & - & - & - & 100 & 100 \\
\hline $\begin{array}{l}\text { D5- O médico/ } \\
\text { enfermeiro lhe dá } \\
\text { tempo suficiente } \\
\text { para você falar sobre } \\
\text { suas preocupaçōes } \\
\text { ou problemas? }\end{array}$ & 60 & 60 & 37 & 37 & 3 & 3 & - & & - & - & 100 & 100 \\
\hline $\begin{array}{l}\text { D6- Você se sente à } \\
\text { vontade contando } \\
\text { as preocupações } \\
\text { ou problemas } \\
\text { relacionados a sua } \\
\text { criança ao médico/ } \\
\text { enfermeiro? }\end{array}$ & 47 & 47 & 49 & 49 & 4 & 4 & - & - & - & - & 100 & 100 \\
\hline $\begin{array}{l}\text { D7- O médico/ } \\
\text { enfermeiro conhece } \\
\text { sua criança mais } \\
\text { como pessoa que } \\
\text { somente como } \\
\text { alguém com um } \\
\text { problema de saúde? }\end{array}$ & 43 & 43 & 52 & 52 & 4 & 4 & - & - & 1 & 1 & 100 & 100 \\
\hline
\end{tabular}




\begin{tabular}{|c|c|c|c|c|c|c|c|c|c|c|c|c|}
\hline \multirow[t]{2}{*}{ D- Longitudinalidade } & \multicolumn{2}{|c|}{$\begin{array}{l}\text { Com certeza } \\
\text { sim }\end{array}$} & \multicolumn{2}{|c|}{$\begin{array}{l}\text { Provavelmente } \\
\text { sim }\end{array}$} & \multicolumn{2}{|c|}{$\begin{array}{c}\text { Provavelmente } \\
\text { não }\end{array}$} & \multicolumn{2}{|c|}{ Não lembro } & \multicolumn{2}{|c|}{$\begin{array}{c}\text { Com certeza } \\
\text { não }\end{array}$} & \multicolumn{2}{|c|}{ TOTAL } \\
\hline & $N$ & $\%$ & $\mathrm{~N}$ & $\%$ & $\mathrm{~N}$ & $\%$ & N & $\%$ & $\mathrm{~N}$ & $\%$ & $\mathrm{~N}$ & $\%$ \\
\hline $\begin{array}{c}\text { D8- O médico/ } \\
\text { enfermeiro conhece } \\
\text { a história clínica de } \\
\text { sua criança? }\end{array}$ & 47 & 47 & 48 & 48 & 5 & 5 & - & - & - & - & 100 & 100 \\
\hline $\begin{array}{l}\text { D9- O médico/ } \\
\text { enfermeiro sabe a } \\
\text { respeito sobre os } \\
\text { medicamentos que } \\
\text { sua criança está } \\
\text { usando? }\end{array}$ & 34 & 34 & 48 & 48 & 18 & 18 & - & - & - & - & 100 & 100 \\
\hline $\begin{array}{l}\text { D10- Você mudaria } \\
\text { de unidade de saúde } \\
\text { para outra se isso } \\
\text { fosse fácil de fazer? }\end{array}$ & 10 & 10 & 30 & 30 & 60 & 60 & - & - & - & - & 100 & 100 \\
\hline $\begin{array}{c}\text { D1 1- Você acha que } \\
\text { o médico/enfermeiro } \\
\text { conhece sua família } \\
\text { bastante? }\end{array}$ & 21 & 21 & 49 & 49 & 30 & 30 & - & - & - & - & 100 & 100 \\
\hline $\begin{array}{c}\text { D12- O médico/ } \\
\text { enfermeiro sabe } \\
\text { quais são problemas } \\
\text { mais importantes } \\
\text { para você e sua } \\
\text { família? }\end{array}$ & 19 & 19 & 62 & 62 & 19 & 19 & - & - & - & - & 100 & 100 \\
\hline $\begin{array}{c}\text { D13- O médico/ } \\
\text { enfermeiro sabe } \\
\text { sobre o trabalho } \\
\text { ou emprego dos } \\
\text { familiares de sua } \\
\text { criança? }\end{array}$ & 17 & 17 & 56 & 56 & 16 & 16 & 1 & 1 & - & - & 100 & 100 \\
\hline $\begin{array}{l}\text { D14- O médico/ } \\
\text { enfermeiro saberia } \\
\text { de alguma forma } \\
\text { se você tivesse } \\
\text { dificuldades em } \\
\text { obter ou pagar } \\
\text { algum medicamento } \\
\text { de sua criança? }\end{array}$ & 18 & 18 & 64 & 64 & 17 & 17 & 1 & 1 & - & - & 100 & 100 \\
\hline
\end{tabular}

Fonte: dados da pesquisa, 2016.

No momento do atributo longitudinalidade é avaliada a relação entre usuário e profissional, no presente estudo observa-se que houve uma boa avaliação por parte dos usuários da ESF, isso demonstra o grau de confiança e interação estabelecido entre o usuário e os profissionais de saúde, podendo ser observado no ítem em que os usuários são questionados se é o mesmo médico ou enfermeiro que Ihe atende na US e $48 \%$ afirmaram que sim e se houvesse possibilidade, se trocariam de unidade $60 \%$ responderam que provavelmente não.

Segundo SILVA, a presença adequada da longitudinalidade é um fator importante para o sistema de saúde, pois geram diagnósticos e tratamentos mais precisos, além da redução dos encaminhamentos desnecessários para especialistas e também permite que as pessoas tenham continuidade do atendimento à saúde ${ }^{15}$. 
No estudo de CHOMATAS, também realizado no município de Curitiba, obteve-se alto índice de satisfação demonstrando que os profissionais de saúde da APS possuem bom vínculo no trabalho com a comunidade ${ }^{14}$. Fato também observado nesta pesquisa, na qual visualizamos que $60 \%$ dos usuários afirmaram que o médico/enfermeiro lhe dá tempo suficiente para falar sobre suas preocupações ou problemas.

\section{COORDENAÇÃO}

O que concerne à coordenação é visualizado na TABELA 5, sendo realizados questionamentos aos pais ou responsáveis se sua criança consultou qualquer tipo de especialista enquanto esteve em acompanhamento com a unidade de saúde.

Tabela 5 - Coordenação

\begin{tabular}{c|c|c|c|c|c|c|c|c|c|c}
\hline E- Coordenação & \multicolumn{2}{c|}{$\begin{array}{c}\text { Com certeza } \\
\text { sim }\end{array}$} & \multicolumn{2}{c|}{$\begin{array}{c}\text { Provavelmente } \\
\text { sim }\end{array}$} & \multicolumn{2}{c|}{ Sim } & \multicolumn{2}{c|}{ Não } & \multicolumn{2}{c}{ TOTAL } \\
\hline $\begin{array}{c}\text { E1- Sua criança } \\
\text { consultou qualquer } \\
\text { tipo de especialista } \\
\text { enquanto ela está em } \\
\text { acompanhamento } \\
\text { com a unidade de } \\
\text { saúde? }\end{array}$ & - & - & - & - & 12 & 12 & 88 & 88 & 100 & 100 \\
\hline $\begin{array}{c}\text { E2- A unidade } \\
\text { de saúde que } \\
\text { encaminhou sua } \\
\text { criança para o serviço } \\
\text { de especialistas? }\end{array}$ & 9 & 75 & 3 & 25 & - & - & - & - & 100 & 100 \\
\hline $\begin{array}{c}\text { E3- O médico da } \\
\text { unidade de saúde } \\
\text { sabe que sua criança } \\
\text { consultou algum } \\
\text { especialista? }\end{array}$ & 6 & 50 & 6 & 50 & - & - & - & - & 100 & 100 \\
\hline $\begin{array}{c}\text { E4- O médico soube da } \\
\text { consulta? }\end{array}$ & 7 & 58 & 5 & 42 & - & - & - & - & 100 & 100 \\
\hline
\end{tabular}

Fonte: dados da pesquisa, 2016.

Pode-se definir como coordenação a articulação entre os diversos serviços e ações de saúde. Essa é uma maneira de dar continuidade por parte dos profissionais no reconhecimento de problemas. Oferta-se ao usuário um conjunto de serviços e informações que respondam a suas necessidades de saúde de forma integrada ${ }^{4,9}$. Em relação aos encaminhamentos à especialistas, $12 \%$ passaram por consultas no serviço de referência, sendo $75 \%$ referenciados pela própria US, demonstrando a relação entre os serviços da rede de atenção à saúde, fazendo com que o usuário possa ser atendido integralmente de acordo com suas necessidades.

\section{SISTEMA DE INFORMAÇÕES}

Referente ao sistema de informações, visualiza-se na TABELA 6. 
Tabela 6 - Coordenação - sistema de informações

\begin{tabular}{c|c|c|c|c|c|c|c|c|c|c}
\hline $\begin{array}{c}\text { F- Coordenação } \\
\text { - sistema de } \\
\text { informaçốes }\end{array}$ & \multicolumn{2}{|c|}{$\begin{array}{c}\text { Com certeza } \\
\text { sim }\end{array}$} & \multicolumn{2}{|c|}{$\begin{array}{c}\text { Provavelmente } \\
\text { sim }\end{array}$} & \multicolumn{2}{c|}{$\begin{array}{c}\text { Provavelmente } \\
\text { não }\end{array}$} & \multicolumn{2}{c|}{ Não lembro } & \multicolumn{2}{c}{ TOTAL } \\
\hline $\begin{array}{c}\text { F1- Quando você leva } \\
\text { sua criança à unidade } \\
\text { de saúde você leva a } \\
\text { carteira de vacinação? }\end{array}$ & 63 & 63 & 37 & 37 & - & - & - & - & 100 & 100 \\
\hline $\begin{array}{c}\text { F2- Quando você } \\
\text { leva sua criança à } \\
\text { unidade de saúde o } \\
\text { prontuário dela está } \\
\text { sempre disponivel na } \\
\text { consulta? }\end{array}$ & 54 & 54 & 46 & 46 & - & - & - & - & 100 & 100 \\
\hline $\begin{array}{c}\text { F3- Você poderia } \\
\text { consultar o prontuário } \\
\text { da sua criança na } \\
\text { unidade de saúde se } \\
\text { você quisesse? }\end{array}$ & 54 & 54 & 45 & 45 & - & - & 1 & 1 & 100 & 100 \\
\hline
\end{tabular}

Fonte: dados da pesquisa, 2016.

Algumas necessidades em saúde podem ser complexas e em geral não são tratadas adequadamente por sistemas de saúde. A inadequação pode não só deixar de atender algumas necessidades, como também ocasionar duplicidade de ações ${ }^{9}$. Em compensação, os atributos da coordenação neste estudo foram bem avaliados, $63 \%$ dos entrevistados levam a carteira de vacinação nas consultas, sendo ela um meio de informação útil no atendimento, $54 \%$ afirmaram que o prontuário da criança está sempre disponível nas consultas e que se precisassem também poderiam ter acesso a ele. Sabemos da importância do sistema informatizado, pois facilita o atendimento e propicia que o profissional consulte o prontuário informatizado do paciente, verifique resultados dos exames laboratoriais, entre outros benefícios que os sistemas de informação proporcionam.

\section{INTEGRALIDADE - SERVIÇOS DISPONIVEIS}

Na TABELA 7 demonstra-se a integralidade dos serviços disponíveis. As perguntas realizadas tiveram relação com os serviços oferecidos pela US.

Tabela 7 - Integralidade - serviços disponíveis

\begin{tabular}{|c|c|c|c|c|c|c|c|c|c|c|}
\hline \multirow[t]{2}{*}{$\begin{array}{l}\text { G- Integralidade- serviços } \\
\text { disponíveis }\end{array}$} & \multicolumn{2}{|c|}{$\begin{array}{l}\text { Com certeza } \\
\text { sim }\end{array}$} & \multicolumn{2}{|c|}{$\begin{array}{l}\text { Provavelmente } \\
\text { sim }\end{array}$} & \multicolumn{2}{|c|}{$\begin{array}{c}\text { Provavelmente } \\
\text { não }\end{array}$} & \multicolumn{2}{|c|}{ Não lembro } & \multicolumn{2}{|c|}{ TOTAL } \\
\hline & $\mathrm{N}$ & $\%$ & $\mathrm{~N}$ & $\%$ & $\mathrm{~N}$ & $\%$ & N & $\%$ & N & $\%$ \\
\hline G1-Vacinas & 90 & 90 & 10 & 10 & - & - & - & - & 100 & 100 \\
\hline $\begin{array}{c}\text { G2- Verificar se sua família } \\
\text { pode participar de algum } \\
\text { programa de assistência } \\
\text { social }\end{array}$ & 74 & 74 & 24 & 24 & 2 & 2 & - & - & 100 & 100 \\
\hline G3- Planejamento familiar & 77 & 77 & 23 & 23 & - & - & 1 & 1 & 100 & 100 \\
\hline
\end{tabular}




\begin{tabular}{|c|c|c|c|c|c|c|c|c|c|c|}
\hline \multirow[t]{2}{*}{$\begin{array}{l}\text { G- Integralidade- serviços } \\
\text { disponiveis }\end{array}$} & \multicolumn{2}{|c|}{$\begin{array}{l}\text { Com certeza } \\
\text { sim }\end{array}$} & \multicolumn{2}{|c|}{$\begin{array}{l}\text { Provavelmente } \\
\text { sim }\end{array}$} & \multicolumn{2}{|c|}{$\begin{array}{c}\text { Provavelmente } \\
\text { não }\end{array}$} & \multicolumn{2}{|c|}{ Não lembro } & \multicolumn{2}{|c|}{ TOTAL } \\
\hline & $N$ & $\%$ & $\mathrm{~N}$ & $\%$ & $\mathrm{~N}$ & $\%$ & N & $\%$ & N & $\%$ \\
\hline $\begin{array}{c}\text { G4- Programa } \\
\text { suplementação nutricional }\end{array}$ & 71 & 71 & 27 & 27 & 1 & 1 & 1 & 1 & 100 & 100 \\
\hline $\begin{array}{l}\text { G5- Aconselhamento } \\
\text { ou tratamento para uso } \\
\text { prejudicial de drogas }\end{array}$ & 65 & 65 & 33 & 33 & 2 & 2 & - & - & 100 & 100 \\
\hline $\begin{array}{l}\text { G6- Aconselhamento de } \\
\text { saúde mental }\end{array}$ & 68 & 68 & 31 & 31 & - & - & 1 & 1 & 100 & 100 \\
\hline $\begin{array}{l}\text { G7- Sutura de um corte que } \\
\text { necessite de pontos }\end{array}$ & 77 & 77 & 22 & 22 & - & - & 1 & 1 & 100 & 100 \\
\hline $\begin{array}{l}\text { G8- Aconselhamento e } \\
\text { solicitação de teste anti-hiv }\end{array}$ & 78 & 78 & 21 & 21 & 1 & 1 & - & - & 100 & 100 \\
\hline $\begin{array}{l}\text { G9- Identificação de } \\
\text { problemas visuais }\end{array}$ & 69 & 69 & 31 & 31 & - & - & - & - & 100 & 100 \\
\hline
\end{tabular}

Fonte: dados da pesquisa, 2016.

Aintegralidade atua como garantia de acesso aos diversos níveis de atenção à saúde, como também os cuidados de que o paciente necessita. A atenção integral depende de uma rede articulada de tal forma que os problemas apresentados pelos indivíduos possam ser abordados para sua melhor resolução ${ }^{9}$. Os serviços disponíveis são compostos por tópicos que são avaliados na APS, o indicador de disponibilidade de vacinas, ou seja, quando perguntamos da disponibilidade de vacinas na ESF para os entrevistados, $90 \%$ responderam que com certeza sim, esta situação é explicada pela existência de vacinas nas unidades de ESF, além de que os pais ou responsáveis se mostraram cientes da importância deste procedimento, colaborando para a promoção e manutenção de sua saúde.

Outros indicadores observados foram o de participação em programas sociais com 74\%, planejamento familiar $77 \%$, Programa suplementação nutricional $71 \%$, o do aconselhamento ou tratamento para uso prejudicial de drogas que apresentou $65 \%$ dos que responderam com certeza sim, possuindo a menor porcentagem dos indicadores, fato que pode ser explicado pela falta de conhecimento dos pacientes sobre este serviço. Aconselhamento de saúde mental $68 \%$, sutura com $77 \%$, aconselhamento e solicitação de teste anti-hiv $78 \%$, identificação de problemas visuais $69 \%$. A análise dos serviços prestados mostrou que os usuários reconhecem claramente a ESF como local para ações em saúde dirigidas à prevenção de agravos e ao controle de saúde.

\section{INTEGRALIDADE - SERVIÇOS PRESTADOS}

No tocante à integralidade dos serviços prestados, encontra-se na TABELA 8.

Tabela 8 - Integralidade - serviços prestados

\begin{tabular}{|c|c|c|c|c|c|c|c|c|c|c|}
\hline \multirow[t]{2}{*}{$\begin{array}{l}\text { H- Integralidade- serviços } \\
\text { prestados }\end{array}$} & \multicolumn{2}{|c|}{$\begin{array}{l}\text { Com certeza } \\
\text { sim }\end{array}$} & \multicolumn{2}{|c|}{$\begin{array}{l}\text { Provavelmente } \\
\text { sim }\end{array}$} & \multicolumn{2}{|c|}{$\begin{array}{c}\text { Provavelmente } \\
\text { não }\end{array}$} & \multicolumn{2}{|c|}{ Não lembro } & \multicolumn{2}{|c|}{ TOTAL } \\
\hline & $\mathrm{N}$ & $\%$ & $\mathrm{~N}$ & $\%$ & $\mathrm{~N}$ & $\%$ & $\mathrm{~N}$ & $\%$ & $\mathrm{~N}$ & $\%$ \\
\hline $\begin{array}{l}\text { H1 - Orientação para manter } \\
\text { sua criança saudável? (sono, } \\
\text { higiene, alimentação) }\end{array}$ & 75 & 75 & 23 & 23 & 2 & 2 & - & - & 100 & 100 \\
\hline H2-Segurança no lar & 60 & 60 & 36 & 36 & 3 & 3 & 1 & 1 & 100 & 100 \\
\hline
\end{tabular}




\begin{tabular}{c|c|c|c|c|c|c|c|c|c}
\hline $\begin{array}{c}\text { H3- Mudanças } \\
\text { no crescimento e } \\
\text { desenvolvimento }\end{array}$ & 72 & 72 & 27 & 27 & 2 & 2 & - & - & 100 \\
\hline $\begin{array}{c}\text { H4- Maneiras de lidar com o } \\
\text { comportamento da criança }\end{array}$ & 67 & 67 & 29 & 29 & 4 & 4 & - & - & 100 \\
\hline $\begin{array}{c}\text { H5- Maneiras de manter sua } \\
\text { criança segura }\end{array}$ & 67 & 67 & 28 & 28 & 5 & 5 & - & - & 100 \\
\hline
\end{tabular}

Fonte: dados da pesquisa, 2016.

Concernente ao atributo Integralidade - Serviços Prestados, foi observado que $75 \%$ dos sujeitos referiram ter tido durante as consultas de puericultura, orientação sobre a saúde, alimentação, higiene ou sono adequado de sua criança, sendo assim, os resultados dessa pesquisa corroboram com FERREIRA, uma vez que as ESF foram identificadas como ponto de partida para o cuidado da criança.

Os entrevistados também foram questionados se houve dicas sobre segurança no lar, $60 \%$ responderam que com certeza sim, $72 \%$ afirmaram que receberam informações sobre mudanças no desenvolvimento infantil, e $67 \%$ relataram que tiveram orientação sobre comportamento da criança e maneiras de mante-lá segura. A relevância da integralidade nos serviços de saúde é caracterizada pela presença dos serviços disponíveis e prestados à população, ofertando os cuidados de atenção na promoção, proteção e recuperação da saúde. Destaca-se que, quando a realização dos serviços prestados é bem efetuada, auxilia também no desenvolvimento da criança ${ }^{8}$.

\section{ORIENTAÇÃO FAMILIAR}

Representa-se TABELA 9 a orientação familiar realizada pelos profissionais de saúde.

Tabela 9 - Orientação Familiar

\begin{tabular}{|c|c|c|c|c|c|c|c|c|c|c|}
\hline \multirow[t]{2}{*}{ I- Orientação familiar } & \multicolumn{2}{|c|}{$\begin{array}{c}\text { Com certeza } \\
\text { sim }\end{array}$} & \multicolumn{2}{|c|}{$\begin{array}{l}\text { Provavelmente } \\
\text { sim }\end{array}$} & \multicolumn{2}{|c|}{$\begin{array}{l}\text { Provavelmente } \\
\text { não }\end{array}$} & \multicolumn{2}{|c|}{ Não lembro } & \multicolumn{2}{|c|}{ TOTAL } \\
\hline & $\mathrm{N}$ & $\%$ & $\mathrm{~N}$ & $\%$ & $\mathrm{~N}$ & $\%$ & N & $\%$ & $\mathrm{~N}$ & $\%$ \\
\hline $\begin{array}{c}\text { I1 - O seu médico / enfermeiro } \\
\text { lhe pergunta sobre suas } \\
\text { opiniões no tratamento da } \\
\text { criança? }\end{array}$ & 47 & 47 & 43 & 43 & 9 & 9 & 1 & 1 & 100 & 100 \\
\hline $\begin{array}{c}\text { I2- O seu médico / enfermeiro } \\
\text { já Ihe perguntou sobre as } \\
\text { doenças que existam em sua } \\
\text { família }\end{array}$ & 44 & 44 & 45 & 45 & 10 & 10 & 1 & 1 & 100 & 100 \\
\hline $\begin{array}{l}\text { I3- O seu médico / enfermeiro } \\
\text { se reuniria com outros } \\
\text { membros da família de sua } \\
\text { criança caso você achasse } \\
\text { necessário? }\end{array}$ & 48 & 48 & 40 & 40 & 10 & 10 & 2 & 2 & 100 & 100 \\
\hline
\end{tabular}

Fonte: dados da pesquisa, 2016.

Visualizou-se que os resultados desse atributo concordam com FRACOLLI, destaca-se que $48 \%$ disseram que com certeza sim o médico/enfermeiro se reuniria com outros membros de sua família caso você achasse necessário para discutir sobre o tratamento da saúde da criança.

O atributo orientação familiar verifica a importância que o profissional de saúde dá para a opinião do cuidador da criança em relação à escolha do tratamento e cuidados da criança, e se o profissional faz perguntas sobre doenças de 
influência genética que ocorrem na família 4 . Observamos que $47 \%$ dos profissionais consultam o cuidador em relação ao melhor tratamento para a criança, empoderando a família para o cuidado e possibilitando melhor efetividade do tratamento, já que este pode se adequar à rotina e as características de cada família.

Em relação ao questionamento sobre o histórico familiar de doenças, $45 \%$ responderam que provavelmente já foram questionados, sendo importante na investigação de síndromes e doenças genéticas, podendo gerar encaminhamentos à especialistas.

\section{ORIENTAÇÃO COMUNITÁRIA}

A realização da orientação comunitária é analisada na TABELA 10.

Tabela 10 - Orientação comunitária

\begin{tabular}{c|c|c|c|c|c|c|c|c|c|c}
\hline J- Orientação comunitária & \multicolumn{2}{|c|}{$\begin{array}{c}\text { Com certeza } \\
\text { sim }\end{array}$} & \multicolumn{2}{|c|}{$\begin{array}{c}\text { Provavelmente } \\
\text { sim }\end{array}$} & \multicolumn{2}{c|}{$\begin{array}{c}\text { Provavelmente } \\
\text { não }\end{array}$} & \multicolumn{2}{|c|}{ Não lembro } & \multicolumn{2}{|c}{ TOTAL } \\
\hline $\begin{array}{c}\text { Ju-Alguém da unidade } \\
\text { de saúde faz visitas } \\
\text { domiciliares? }\end{array}$ & 56 & 56 & 33 & 33 & 9 & 9 & 2 & 2 & 100 & 100 \\
\hline $\begin{array}{c}\text { J2-A equipe de saúde } \\
\text { conhece os problemas de } \\
\text { saúde importantes de sua } \\
\text { vizinhança? }\end{array}$ & 40 & 40 & 46 & 46 & 14 & 14 & - & - & 100 & 100 \\
\hline $\begin{array}{c}\text { J3-A equipe de saúde faz } \\
\text { pesquisas na comunidade } \\
\text { para identificar os } \\
\text { problemas de saúde que eles } \\
\text { deveriam conhecer? }\end{array}$ & 36 & 36 & 47 & 47 & 16 & 16 & 1 & 1 & 100 & 100 \\
\hline $\begin{array}{c}\text { J4- Convida membros da } \\
\text { familia a participar do } \\
\text { conselho local de saúde? }\end{array}$ & 38 & 38 & 49 & 49 & 12 & 12 & 1 & 1 & 100 & 100 \\
\hline
\end{tabular}

Fonte: dados da pesquisa, 2016.

O atributo Orientação Comunitária apresenta em seus itens se os profissionais fazem a visita domiciliar, prática diferencial da ESF, tendo $56 \%$ dos entrevistados afirmando que receberam visitas. Nesta atividade se fazem pesquisas sobre o processo saúde doença da população e do contexto de vida e saúde das famílias ${ }^{6}$. Como é observado nesse estudo e no de FRACOLLI e FERREIRA, apenas $38 \%$ dos entrevistados referem que com certeza sim, recebem convite para participar do conselho local de saúde, no entanto, os profissionais que trabalham na ESF, por sua proximidade com a população, devem estimular a participação social e o controle social por parte da comunidade.

Os resultados mostram que de forma geral as duas US possuem uma boa avaliação para APS, sendo evidenciando que os cuidados primários contribuem de forma decisiva para a melhoria dos indicadores de saúde. As Ferramentas de Avaliação são apropriadas para medir a realização dos atributos, pois fornecem informações sobre os elementos de estrutura e processo. Isso também inclui conhecimento sobre o foco da US, as características do paciente e os serviços disponíveis no local ${ }^{16}$.

\section{Considerações Finais}

Conclui-se que nas duas unidades ESF, os pais ou cuidadores fizeram uma boa avaliação dos atributos da APS, apresentando um bom grau de afiliação com serviço, considerando a acessibilidade das US, como principal estratégia para organização do trabalho, destaca-se que a integralidade dos serviços disponíveis e serviços prestados ao público infantil permitiu o estreitamento de relacionamento entre profissional de saúde e o usuário. 
Ressalta-se que a percepção dos usuários em questão, quanto ao serviço da APS, contribui para subsidiar melhorias na assistência prestada nas US e aumentar a qualificação dos profissionais de saúde. 0 trabalho realizado permite identificar com mais clareza o efetivo impacto que o cuidado prestado na APS possa estar trazendo a esta população do estudo. Além de ser importante para que os profissionais da saúde busquem novos métodos e alternativas para definir estratégias de promoção e prevenção.

No entanto, a pesquisa possui limitações no que diz respeito ser um estudo local, abrangendo apenas a perspectiva dos cuidadores de crianças menores de dois anos que utilizam o serviço de duas ESF, podendo, nesse caso, não representar a complexidade e a totalidade do serviço de saúde da criança disponível na APS do município de Curitiba-PR.

Espera-se que possa impulsionar outros municípios a elaborarem estratégias e realizar a avaliação de outras realidades, visto que ele foi importante não somente para o conhecimento estatístico da satisfação do usuário, mas para contribuir para qualificação das ações voltadas para APS do município de Curitiba.

Os resultados devem levar a sensibilização dos gestores sobre a importância desta área estratégica da saúde da criança, reorganizando a atenção à saúde e as linhas de cuidado para esta clientela através das tecnologias de cuidado, concordando e sempre em consonância com o que é preconizado pelo Ministério da Saúde.

\section{Referências}

1. MATTA GC, MOROSINI MVG. A atenção primária a saúde. Dicionário da educação profissional em saúde, 2012. [acesso em 2015 mar. 10]. Disponível em: Disponível em: www.epsjv.fiocruz.br

2. BRASIL. Ministério da Saúde. Política Nacional da Atenção Básica. Secretaria de Atenção à Saúde. Departamento de Atenção Básica. [Internet]. Brasília: Ministério da Saúde; 2012. [acesso em 2016 ago. 16]. Disponível em: http://189.28.128.100/dab/docs/publicacoes/geral/pnab.pdf

3. MOREIRA MEL, GOLDANI MZ. A criança é o pai do homem: novos desafios para a área de saúde da criança. Ciência e saúde coletiva, v.15, n.2, p. 321-327, Rio de Janeiro, 2010

4. FRACOLLI LA, MURAMATSU MJ, GOMES MFP, NABAO FRZ. Avaliação dos atributos da Atenção Primária à Saúde num município do interior do Estado de São Paulo - Brasil. Ver. O Mundo da Saúde, v. 39, n.1, São Paulo, 2015.

5. MENDES EV. O cuidado das condições crônicas na atenção primária à saúde: 0 imperativo da consolidação da estratégia da saúde da família. Eugênio Vilaça Mendes. [internet]. Brasília: Organização Pan-Americana da Saúde, 2012. [acesso em 2016 ago. 16]. Disponível em: http://bvsms.saude.gov.br/bvs/publicacoes/cuidado_condicoes_atencao_ primaria_saude.pdf

6. BRASIL. Ministério da Saúde. Secretaria de Atenção em Saúde. Departamento de Atenção Básica. Manual do instrumento de avaliação da atenção primária à saúde: primary care assessment tool pcatool - Brasil. Ministério da Saúde, Secretaria de Atenção em Saúde, Departamento de Atenção Básica. [Internet]- Brasília: Ministério da Saúde, 2010. [acesso em 2016 ago. 16]. Disponível em: http://bvsms.saude.gov.br/bvs/publicacoes/manual_avaliacao_pcatool_brasil.pdf

7. DAMASCENO SS. Avaliação da orientação à atenção primária à saúde da criança. 2014.140f. [Dissertação]. Centro de ciências da saúde, Universidade Federal da Paraíba, 2014. .[acesso em 2016 ago.16]. Disponível em: http://tede. biblioteca.ufpb.br:8080/handle/tede/5146

8. FERREIRA TLS, COSTA ICC, ANDRADE FB. Avaliação do atributo integralidade em serviços de puericultura na atenção primária à saúde. Revista Ciência Plural. V. , N. 1, P. 22-29, 2015.

9. OLIVEIRA MA, PEREIRA IC. Atributos essenciais da Atenção Primária e a Estratégia Saúde da Família. Rev Bras Enferm. V. esp, N.66, P. 158-164, 2013.

10. OLIVA ACD, MOURA CMR, LIMA CA, COSTA FM, ROCHA JFD. Avaliação dos atributos do cuidado primário de saúde na perspectiva do usuário. Rev. Uniabeu Belford Roxo. V. 8, n. 18, 2015.

11. BRAZ JC. Avaliação da atenção às crianças menores de um ano na Estratégia Saúde da Família em um município da Bahia, sob a ótica dos cuidadores. 2012. 83f. [Dissertação]. Escola de Enfermagem de Ribeirão Preto, Universidade de São Paulo, Ribeirão Preto, 2012.[acesso em 2016 ago.16]. Disponível em: http://www.teses.usp.br/teses/ disponiveis/22/22133/tde-15012013-113853/pt-br.php

12. GARUZI M, ACHITTI COM, SATO CA, ROCHAAS, SPAGNUOLO RS. Acolhimento na Estratégia Saúde da Família: revisão integrativa. Rev Panam Salud Publica3, V. 5, N.2, 144-9.2014. 
13. HAGGERTY JL, LEVESQUE JF, SANTOR DA, BURGE F, BEAULIEU C, BOUHARAOUI F, et. al. Accessibility from the Patient Perspective: Comparison of Primary Healthcare Evaluation Instruments. Healthc Policy. Dec; 7(Spec Issue): 94-107. 2011.

14. CHOMATAS E, VIGO A, MARTY I, HAUSER L, HARZHEIM, E. Avaliação da presença e extensão dos atributos da atenção primária em Curitiba. Rev Bras Med Fam Comunidade, v 29, n. 8, P. 294-303, Rio de Janeiro, 2013.

15. SILVA CSO, FONSECAADG, SOUZA LPS, SIQUEIRA LG, BELASCO AGS, BARBOSA DA. Integralidade e Atenção Primária à Saúde: avaliação sob a ótica dos usuários. Ciência \& Saúde Coletiva, V. 19, N.11, P.4407-4415, 2014.

16. Johns Hopkins University. Primary Care Assessment Tools [Internet]. Baltimore; 2016 [acesso em 2016 ago. 16]. Disponível em: http://www.jhsph.edu/research/centers-and-institutes/johns-hopkins-primary-care-policy-center/pca_tools.html

\author{
Bianca Fontana Aguiar \\ Endereço para correspondência - Rua: Carlos de Laet, $n^{\circ} 241$, \\ Bairro: Oficinas, CEP: 84035440, Ponta Grossa, PR, Brasil. \\ E-mail: biancafoguiar@hotmail.com \\ Lattes: http://lattes.cnpq.br/9180351711874168 \\ Noeli Maria Rodrigues Alves Santos Hack - neli.hack@fpp.edu.br \\ Leandro Rozin - leandrorozin@hotmail.com \\ Enviado em 27 de junho de 2016. \\ Aceito em 18 de agosto de 2016.
}

